\title{
IX. SYNAPSES, CYTOKINES (CK) AND LONG-TERM \\ MEMORY (LTM): AN INTERDISCIPLINARY \\ LOOK AT HOW PSYCHOANALYSIS MIGHT ACTIVATE \\ LEARNING VIA ITS \\ EFFECTS ON EMOTIONAL ATTENTION1
}

\author{
FRED M. LEVIN
}

\begin{abstract}
"Weeds grow and animals slip about in the night where no man dares to hunt them down."

Loren Eiseley (1971). The Night Country, p. 3
\end{abstract}

I begin with a quote from Loren Eiseley about the darker side of mental life because despite all of our increasing knowledge of $\mathrm{mind} /$ brain we cannot completely mitigate human suffering. Obviously, a lot depends upon how any new knowledge we acquire would be applied. My hope is that our latest neuropsychoanalytic insights will lead to the improved application of psychoanalysis clinically, especially to matters of the human heart, which Eiseley wrote about so movingly.

Eiseley was an only child, the son of a poor deaf mother during an age when deaf people were ostracized as "deaf and dumb". He lost his father early in his life, was picked upon frequently by playmates, eventually leaving home in his youth to ride freight trains across the U.S.A. during the Great Depression. His vagabond life lasted until he found an island of hope in the night country of his mind. Against all odds, Eiseley ultimately became the distinguished Chair of Anthropology at the University of Pennsylvania, and one of America's great writers! ${ }^{2}$ He had learned to collaborate with feared others, but he never forgot the inner darkness.

From time to time in what follows I intersperse the dry technical details on neural networks, memory and learning with a completely imagi-

${ }^{1}$ The author wishes to thank Jean Carney, Nancy Desert, John Gedo, Eric Gillett, Masao Ito, Thomas Launey, Stanley Palombo, Michael Posner, Arnold Wilson, and Kasuhiko Yamaguchi for their useful suggestions, while he personally takes full responsibility for the final product, and inevitable mistakes.

${ }^{2}$ On the dust jacket of Eiseley's (1971) book, Ray Bradbury sees Eiseley's writings as likely to "linger for the next century". 
nary psychoanalytic interchange between myself and Loren Eiseley! ${ }^{3}$ This should remind us of the human side of my story.

Psychoanalysis, psychology, and neuroscience each contribute to learning theory. My paper discusses some of the details that make this so, and aims towards a unified theory of learning, but one based on interdisciplinary studies which I believe are significantly changing psychoanalytic theory and practice, and impacting as well on neuroscience and psychology.

There will be three parts to my paper: A summary of the Neuroimmune Network (NIN), including the subjects of cytokines (CK) and synapses; an account of long-term memory (LTM); and a postscript on interdisciplinary research.

The key to Horst Ibelgauft's webpage (1999) on the NIN (Levin 2002) and to this paper is the role of cytokines on synaptic plasticity, via their ability to activate and inactivate genes. To quote Abel, Martin, Bartsch and Kandel (1998): "The neuronal activation of genes means simply that the molecular basis of memory may hinge on the balance between chemical factors that establish versus those which inhibit synaptic plasticity" (Abel, Martin, Bartsch \& Kandel 1998, p. 338).

As I indicate in the abstract, the novel idea of this paper is that when, under the influence of a psychoanalysis, new opportunities for learning occur, this can reasonably be ascribed to the activation and creation of novel synapses, produced simultaneously with the psychoanalytic maneuvers. First the new synapses help form novel neural networks in the brain of that analytic patient; later these networks will lead to new LTM, that is, new learning. In what follows I shall attempt to eliminate doubt about the truth of these assertions.

For a long time the Holy Grail of neuroscience has been understanding the cellular and molecular chemistry of memory and learning, particularly the synaptic matrix in which CK act. I believe that psychoanalysts can participate in this quest, along with brain scientists, through the search for apt mind/brain correspondences (Solms 1999). Such correspondences are required for the creation of comprehensive evidencebased learning theories that cross disciplinary boundaries. Therefore, in the second part of this paper I briefly revisit my earlier research into

${ }^{3}$ The reader should not confuse my speculations about the imaginary Eiseley, later in this text, with the real man I introduced on page 1. As noted, they are merely imaginative speculations to illustrate various points about psychoanalysis, and any similarity to the real Eiseley is purely coincidental. 
how psychoanalysis facilitates learning readiness (Levin 1991, 1997, 2003). Because our theories and our techniques are inseparable we owe it to our patients to develop both as carefully and objectively as possible. ${ }^{4}$

Learning is an emotional phenomenon, that is, a highly motivated action which satisfies vital needs, and which has its affective origins deep within the brain; it is not a receptive passivity. That learning involves a core emotional self is particularly important because there is reason to believe that modern cognitive neuroscience approaches to learning are seriously deficient in two ways: 1. they tend to downplay emotions because feeling states can be hard to qualify and quantify experimentally, and 2. they often restrict attention to those brain structures which can be seen on scans, which means that deep structures such as the periaqueductal gray (PAG) and the hypothalamus within the brain's core tend to get short shrift. Because of the dominance of cognitive theories of learning and memory we need to learn more about how emotions contribute to the sense of self and related learning. Obviously, emotions and their substrates deserve our attention.

The biology of learning and memory involves various levels of brain organization. A major problem in discussing brain is that going deeply into such subjects as synapses, ion channels, chemical signaling, receptors, second messengers, etc. is very likely to lose the attention of many people. Yet we cannot avoid such considerations if our goal is to set the stage for a serious neuropsychoanalytic consideration of learning and memory, and particularly if we wish to comprehend the complex role of CK.

From a cognitive perspective memory comes in at least two flavors (explicit and implicit), each further dividing into short vs. long-term forms. Explicit memory involves the medial temporal lobe, (including of course the hippocampuses and related structures), while implicit memory involves the cerebellum, basal ganglia, and the amygdalas. In parts I. and II. I will attempt to review the neuroscientific basis for learning and memory.

${ }^{4}$ If one asks how it matters for our understanding of psychodynamics that the mechanisms discussed in this paper and not others are operating, the answer is as follows: reliable treatments, like reliable prediction in any science, rest on accurate understandings, and these can only be discovered by clinical and laboratory experimentation. Proper scientific technique and related theory are based upon various kinds of hard evidence, and many generations of such observations and correlations will be required for real progress to occur. 
Psychoanalytically-oriented research in the area of learning and memory has benefited greatly from the work of large number of scholars, a sample of which I wish to mention now: the late Niels Lassen (on working memory), Michael Posner and Tim Shallice (on executive control), Jaak Panksepp (on core affective systems), John Gedo (on the hierarchical-developmental model), Arnold Modell (on the holding environment), Howard Shevrin (on subliminal perception), Colwyn Trevarthen (on mother-infant interaction), Merton Gill, Karl Pribram, Karen Kaplan-Solms and Mark Solms (on mind-brain correlations), and Eric Kandel and Masao Ito (on synaptic mechanisms, including those of the cerebellum) (Levin 2003; also see Panksepp 2000, 2001).

Standing on the shoulders of these scholars and many others, a picture is slowly emerging regarding how the different levels of mind/brain organization are integrated. For example, we know now that during psychoanalysis learning-readiness is created by the analyst's provision of a proper holding environment, especially as this invites the anlysand's sense of safety and spontaneity. But as Fonagy (1998) has pointed out, what gets learned within any psychoanalysis is more than the consequence of interpretations per se,; analytic learning obviously involves conditioning (procedural learning) as well, so that in the final result, both emotion and insight play critical roles. And if learning is involved, obviously, clearly changes in synaptic plasticity must be stimulated by the analytic method. Or, putting it differently, it seems likely that the learning known to occur during analysis has a basis in mind and brain, and this is likely to involve changes in the plasticity of neurons working within a neural network.

In referring to spontaneity I am considering two aspects of psychoanalysis: 1. the patient's free associations in the context of a trusting (i.e. safe) relationship with his or her analyst, and 2. his or her spontaneous transference enactments. What is important is that it seems to be the spontaneity that activates working memory for those subjects that are remembered or enacted in treatment (Levin 1997). Moreover, when these memories and enactments are interpreted to the patient they begin to effect the patient's response patterns, and ultimately enter emotional attention more pervasively. I am describing a process of learning via emotional attention. This learning occurs whether or not the important

${ }^{5}$ This is not meant to depreciate interpretations within analysis, but rather to elevate the role of analytic associative learning (conditioning) that can profoundly alter mind/brain organization and personal adaptive functioning. 
analytically-observed behavioral or affective patterns ever actually enter the patient's or analyst's consciousness: this follows from what we know about subliminal perception, and its result is knowledge without awareness (see Levin 1997, 2003 for review of Shevrin's work on this subject).

But even more important, the emotional attention and the learning stimulated within a psychoanalysis obviously have a direct relationship to the neurochemical changes occurring within the brain during such states. I will be arguing that the psychological and the neurochemical changes associated with learning are dependent concomitants. I believe that what Freud called "the drive organization of memory" was his intuitively correct depiction of an adaptive learning process ${ }^{6}$ in which synaptic plasticity is altered by affective experience (i.e. needs). This latter viewpoint, however, alters psychoanalytic thinking in a revolutionary way.

I use the expression emotional attention for precise reasons. Emotional because I feel that what first appears as a novel neural learning network, ultimately becomes new long-term memory (LTM). And the novel neural network starts from our being moved by the emotional significance of particular events, as well as from our multiple emotional needs, e.g. for companionship, affection, novelty, and so forth. It is likely that our emotionality is what becomes the trigger for new learning, and for the mind/brain changes that support it (see below). And regarding the phrase emotional attention I use the word attention rather than the word consciousness because, as I noted, many important observations enter into our thinking subliminally.

I shall report in this paper upon details of the chemical and neurophysiological changes associated with neural network formation. Our ultimate goal, of course, is to connect, as much as possible, psychoanalytic learning and those factors contributing to new LTM (Levin 1991, 1995, 1997, 2002). My intuition is that the key to understanding learning within psycho-analysis lies in linking two approaches: 1. those practical procedures within psychoanalysis that invite the activation of working memory and emotional attention, and 2. the psychology of emotional attention and our growing understanding of how insights relate to changes in synaptic plasticity chemically, especially via their effects on CK-mediated gene activation or its matrix.

\footnotetext{
${ }^{6}$ Thanks to John Gedo for this observation (personal communication).
} 
Finally, in the third part of this paper I make some general remarks about the current profusion of schools within psychoanalysis. In my opinion this reflects interdisciplinary and collaborative efforts which are currently informing psychoanalysis with the viewpoints of various specialized areas of knowledge; but, in addition, they are also helping establish several new fields, and not just schools. For example, in the case of efforts to bridge psychoanalysis and neuroscience, they have created neuro-psychoanalysis. This represents a critical paradigm shift in our field, not merely an adjustment of psychoanalytic theory.

\section{THE NEUROIMMUNE NETWORK AND IMMUNITY}

Horst Ibelgauft (1999) at the University of Munich has created a Cytokines $O$ nline $P$ athfinder Encyclopedia (COPE), containing over fifteen thousand scientific references and 50,000 hyperlinks to the thousands of CK listed. He makes clear that the central nervous system (CNS) and the immune system (IS) (including the neuroendocrine system) essentially constitute a single, integrated overarching system, the neuroimmune network (NIN). His conclusion is based upon evidence such as the following: 1. "Neural targets controlling thermogenesis, behavior, sleep, and mood are affected by the so-called pro-inflammatory CK [namely, interleukin 1 (IL-1), interleukin 6 (IL-6), and tumor necrosis factor alfa (TNFalpha)] which are released by macrophages and monocytes during infection"; 2. within the CNS "production of CK has been detected as a result of brain injury, during viral and bacterial infections, and in neurodegenerative processes." In other words, communication between the nervous system and the soma is two way (Ibelgauft, cited in Levin 2002). Moreover, 3. research on the details of synaptic transmission confirms Ibelgauft's conclusion CK play a critical role (for details see Abel, Martin, Bartsch \& Kandel 1998); and 4. there are structural chemical similarities between the CNS and IS, for example, Agrin, an aggregating protein, is expressed in lymphocytes (where it plays a role in $\mathrm{T}$ cell signaling and lipid raft formation, the latter subject mentioned below), as well as in neuronal signaling, and even in the functionality of the myoneural junction (Khan, Bose, Yam, Solski \& Rupp 2001).

So what are CK? They are "a diverse group of soluble proteins and peptides which act as humoral regulators at nano- to picomolar concentrations... and which act on a wider spectrum of target cells than do hormones" (Ibelgauft 1999, section on cytokines, p. 2). "CK [are pro- 
duced by] cells of the immune system, by glial cells, neurons, and by skin cells" (Levin 2002). You will recall that skin cells and neurons begin as neuroectoderm, that is, they are embryologically related to each other.

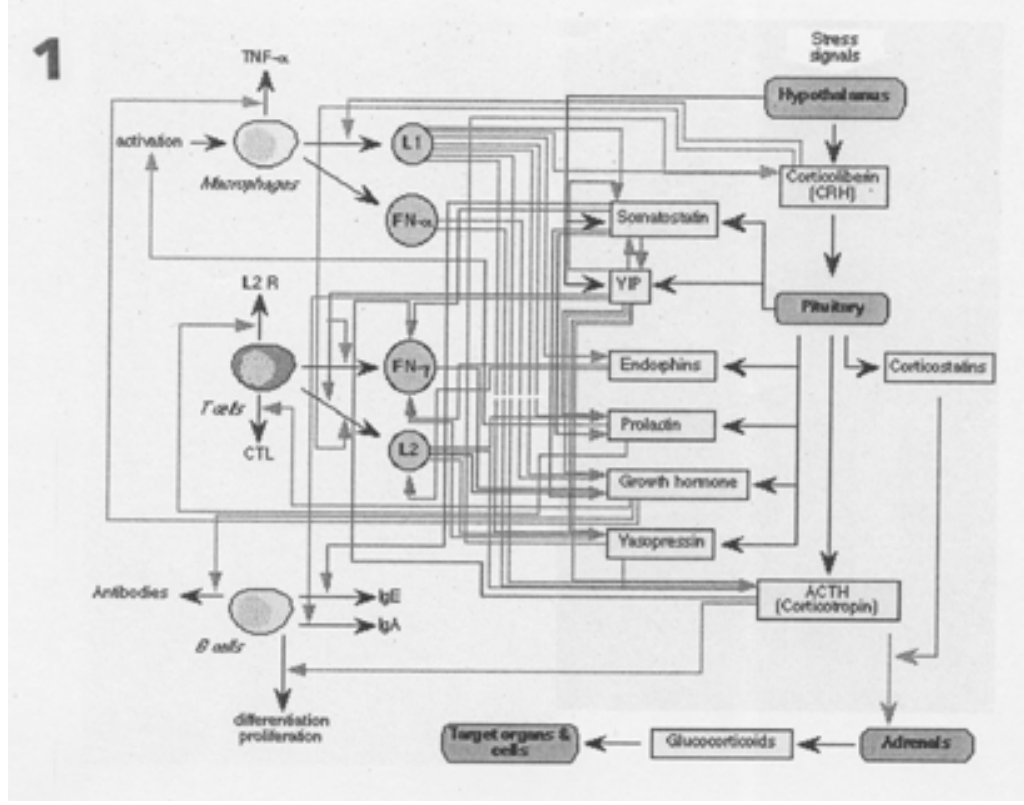

Figure 1

Figure 1 (from Ibelgauft 1999) presents a simplified scheme of the hypothalamic-pituitary axis (HPA).

According to Maier and Watkins (1998), there are at least three pathways between the periphery and the brain. Unidirectional pathways include Pathways A and B. Pathway A is the autonomic nervous system, the sympathetic part of which enervates immune organs. In Pathway A, catecholamines (largely epinephrine and norepinephrine [NE]) are released from sympathetic nerves controlling various organs (e.g. the spleen, thymus, etc.).

Pathway B is the hypothalamic-pituitary axis (HPA). In Pathway B various releasing factors (e.g. corticotropin releasing factor [CRF] whose source is the hypothalamus) enter into the bloodstream heading from hypothalamus to pituitary, which in response releases various hormones on demand (e.g. adreno-corticotropin releasing hormone, or 
$\mathrm{ACTH})$. These pituitary hormones then travel via the blood stream and effect both the peripheral endocrine organs (e.g. the adrenal cortex, which then releases cortisol) and the immune system.

I will jump to the bidirectional immune pathways between the brain and the periphery. One arm of Pathway $\mathrm{C}$ is composed of immune cells (i.e. macrophages and other cells) that releases IL-1 (as an example) which can stimulate sensory paraganglia of the vagus nerve, which in turn carries impulses to the nucleus tractus solitarius (NTS) and the area postrema (AP) in the brain-stem. We do not often think of the vagus nerve as having a sensory component but it does. A neural cascade then occurs which at least theoretically could result in the release into the blood of CK such as IL-1. There is some debate, however, whether any of this blood born IL-1 actually reaches the brain itself or crosses the relatively impervious blood-brain barrier. Thus, possibly more important for CNS CK production is a second arm of pathway $C$ which starts as does the first in the vagus nerve, reaches the NTS and $A P$, but then heads into two different brain areas, the hippocampus and the hypothalamus, wherein $I L-1$ is generated directly within the brain (Levin 2002).

Once so generated in the brain, CK (or the IL-1 in our example) are capable of interacting with any neuron that carries the appropriate CK receptors. Any of three further different reactions then become possible. One is simple neurotransmission, i.e. the generation of a postsynaptic depolarization. Second, there could be a response specific to the CK involved; for example, in the case of IL-1, there would be a proinflammatory response, namely sickness reactions, fever, fatigue, anorexia, etc.

The third possibility is the induction of gene transcription and translation (see below), ${ }^{7}$ which obviously can profoundly effect memory and learning (Levin 2002). CK are thus part of the mechanism for LTM formation, which I intend to review in Part II.

${ }^{7}$ The precise meanings of the technical terms "transcription", "translation", and "copying" within genetics are not obvious, but may be distinguished from each other as follows: transcription refers to the copying of DNA into messenger RNA (mRNA); translation refers to copying RNA code into related proteins (which then express gene functions); incidentally, copying refers to the creation of new forms of RNA from other RNA (for example, under the influence of RNA-dependent RNA polymerase) (Ahlquist 2002, p. 1270). 


\section{1. KINDS OF IMMUNITY}

Some will appreciate a brief review of immunity to place the subsequent discussion of $\mathrm{CK}$ research in a more familiar context. In a recent review of the subject (Levin 2002) I cite Maier and Watkin's (1998) description of immunity as follows: "There are two kinds of immunity, either specific or nonspecific. The former is also called 'humoral' and is slow; the latter is also called 'cellular' and is relatively rapid. Both responses are secondary to the detection of foreign invaders, either viral, bacterial, or toxic-chemical, but they follow distinctly different pathways."

Nonspecific cellular immunity occurs within 1-2 hours of the invasion, and depends upon phagocytes such as macrophages or neutrophils. Later, when I summarize specific immunity, I will note the role of macrophages in that process as well, where they serve as antigen presenting cells (APCs); however, in the present discussion of rapid, nonspecific immune response the goal is three fold: 1 . the nonspecific recognition of foreign material (antigen, none self, "stressor", "toxin"), 2. the transfer of this material into phagocytes ("eating cells"), and 3. its destruction by various intracellular means, including exposure to enzymes, nitric oxide, and/or various CK (e.g. IL-1, IL-6, TNFalpha, etc.). These defensive proteins and other transmitters create the so-called inflammatory reaction which further attracts other immune cells.

Specific humoral immunity is more complex, generates antibodies, and takes 3-5 days because a number of cell reproductive cycles are required to create enough of those cells making the specific antibody targeted at particular antigenic stimuli. First the foreign substance (antigen) is engulfed within the APCs. Parts of the invader are then moved to the surface of the APC. This display of parts is recognized by T cells which carry appropriate surface receptor sites. Recognition involves a binding between the $\mathrm{T}$ cell receptor site and the displayed antigenic part, a process that stimulates the $\mathrm{T}$ cell to differentiate and proliferate, increasing the number of $\mathrm{T}$ cells which can then target the specific antigen (invader).

Some $\mathrm{T}$ cells are cytotoxic, and kill specific antigen producing cells. Others are T-helper cells, which help a separate class of B cells expand their number, and begin to detect antigen, differentiate, and proliferate just as the original $\mathrm{T}$ cells did, thus joining the battle against antigen. From our point of view, what is important is that the substances secreted by the APC which assist their performing complex antigen site 
matching and intercellular communication among the T-helper cells, and the B cells, are basically CK (e.g. the interleukins already mentioned).

\section{2. CK AND SYNAPTIC PLASTICITY}

Since CK are involved in gene activation it will help to define the processes of gene transcription and translation. Transcription involves forming messenger RNA (mRNA) from DNA, a process mediated by specific RNA polymerases active at the ribosomes (Ibelgauft 1999, section on Gene expression, p. 1). The second step, translation, involves mRNA being translated into specific proteins which express functions for particular genes.

As we shall see, many complex factors effect gene expression in addition to the CK I am highlighting in this paper (Levin 2002). For example, all genes have promotor regions which contain special DNA that regulates transcription and processing of the genetic codes. Transcription factors which aggregate in the promotor regions ${ }^{8}$ are referred to as response elements. Some of these transcription factors are required for the transcription of all genes, but others are important primarily or exclusively for special classes of genes. One subgroup of transcription factors or so-called nuclear receptors is called early response genes (ERGs). We will return to ERGs later when we consider the cascade leading to LTM, but first I need to describe the synapse and types of memory.

\section{3. THE SYNAPSE ${ }^{9}$ AND PRIMING OF PLASTICTY}

Everyone knows that synapses are the spaces or clefts into which neurotransmitters are released, thus providing chemical contact between

${ }^{8}$ Within the promotor region are docking components where the many transcription factors attach to each other and the DNA, which enable RNA polymerase to align itself next to the gene which is to be replicated.

${ }^{9}$ One complication of the learning picture presented is that although previously thought impossible, it is now known that new neurons can appear in the brain in various locations. Primate research has shown, for example, that prefrontal, inferior temporal, and posterior parietal cortex is important for cognitive functions, and these three zones demonstrate new neurons that originate in the subventricular zone and migrate through the white matter to the neocortex, where they extend axons. This continues throughout adulthood (see Gould, Reeves, Graziano \& Gross 1999). Unfortunately, this aspect of learning cannot be covered in this paper because of space and knowledge limitations. 
neurons. These transmitters are released from presynaptic vesicles, traverse the synaptic cleft and attach themselves to receptor sites on the postsynaptic membrane (that is, the membrane of the second neuron). This usually allows the first neuron to depolarize the second neuron and continue a nervous excitation wave in a particular direction. ${ }^{10}$ There may be as many as a million million synapses within the CNS. What may not be readily appreciated, however, is that the actual structure and functional relationships of synapses belie the simple description just given! For one, synapses do not just preexist, but are creatable on emotional demand by the activity of cytokines, that is, in relation to the drive towards learning. Second, there is evidence that several proteins are complex regulators of presynaptic plasticity, effecting the three basic steps of neurotransmitter relase, as follows: Rab3A and RIM appear to be involved in vesicle docking, Munc 13 is required for priming, and synaptotagmin effects vesicle fusion (Dobrunz \& Garner 2002). As these authors put it, Schoch et al. (2002) and Castillo et al. (2002) have shown that 'the presynaptic 'bouton' is filled with synaptic vesicles loaded with neurotransmitters. After a priming step these vesicles dock at a specialized region of the presynaptic plasma membrane known as the active zone. Electrical impulses arriving at the active zone trigger the fusion of the synaptic vesicles with the plasma membrane, releasing their neurotransmitter into the synaptic cleft" (Dobrunz \& Garner 2002, p. 277).

Let me take a brief pause. If I imagine Loren Eiseley as my patient in psycho-analytic treatment, I believe the critical issue for him would be whether or not I would be carefully listening to him and remembering what he has told me. Only then would he be reassured that he exists for me, i.e. in an important emotional sense. My hunch is that this kind

${ }^{10}$ Another major complication of this simplified picture is that evidence is accumulating that there are also retrograde messengers informing the presynaptic membrane of events in the postsynaptic membrane, controlling presynaptic membrane neurotransmitter vesicle formation and release. Nitric oxide may play a role here, and arachidonic acid (Bliss 1988, p. 88, see also Linden \& Routtenberg 1989, especially Fig. 2, p. 289). In addition, endogenous cannabinoids can function as retrograde messengers activating $\mathrm{CB} 1$ receptors on the presynaptic side, suppressing neurotransmitter release. CB1 receptors are "one of the most abundant ... in the brain" expressed at high levels in the hippocampus, cortex, cerebellum, and basal ganglia, accounting for the striking effects of cannabinoids on memory and cognition (Wilson \& Nicoll 2002, p. 678). Also, another retrograde signaling pathway involves EphB receptor-Ephrin B ligand interaction in hippocampal mossy fiberCA3 synapses (Contractor et al. 2002). 
of emotional awareness or attention is what serves as a decisive trigger for the induction of new synapses and the use of these in the service of new learning. Whether we are seen, heard, appreciated, and especially loved and respected, is never neutral.

Emotional attention in psychoanalysis may be particularly useful because it feels to us as if it is not guided, that is, it is something felt to be independent of outside agency and thus of immediate interest to the self. In spontaneous activity emotional attention and executive functions seem less in play than they would be otherwise; and perhaps that is why this kind of attention feels important, that is, it seems at time particularly salient for our survival. Selective attention may therefore be a precursor to emotional attention. Csikszentmihalyi argued long ago (1975), and I agree, that emotional attention plays a central role in learning, especially when it falls in some optimal effective range. ${ }^{11}$

Returning to synapses, it is obvious as we make our way through the very dense subject of what happens in the synaptic cleft, as well as at the pre- and postsynaptic membrane levels, that there are many factors which influence synaptic weights and the related learning in networks of neurons. Exploring this complicatedness of synapses is important, however, if we are to understand CK activity within the context of neural transmission, memory and emotional learning. It will therefore help to consider the categories of memory and say something as well about receptors. ${ }^{12}$

As I noted in my introduction, it appears unanimous that there are at least the two major types of memory, implicit and explicit (sometimes called procedural and declarative), at least two kinds of receptors (AMPA or NMDA receptors ${ }^{13}$ ), and the memory types divide neatly anatomically (for explicit memory - lateral temporal lobe and hippocampal vs. for implicit memory - the cerebellum, basal ganglia, and amygdala's). The time course of activity of these two memory systems is also different (in the case of explicit memory, multiple repetitions seem required for learning, and memories are read out by merely asking

${ }^{11}$ Jean Carney, personal communication.

${ }^{12}$ Squire divides memory into declarative (explicit) and non-declarative (implicit); the latter also being called procedural. Explicit involves memory for facts and events; implicit involves memory for skills, priming, simple classical conditioning, and non-associative learning (Squire 1998, p. 62).

${ }^{13}$ NMDA and AMPA receptors are two glutamate receptor families, glutamate (glutamic acid) being the major excitatory neurotransmitter in the brain (Bliss 1998, p. 88-89). 
conscious questions. Nonconscious or unconscious methods can also result in or tap explicit memory. In the case of implicit memory, however, learning usually occurs with a smaller number of passes, ${ }^{14}$ and recall usually obtained by priming, i.e. without consciousness being required). Children do as well as adults in the case of implicit learning.

Another time out. My hypothetical treatment with Eiseley would involve both explicit and implicit memory systems. The implicit system would especially be involved whenever I would prime his memory by reminding him of things he told me earlier, and which might be related to his here and now feelings. For example, imagine that he was talking in the previous session about people who interrupt him with stupid comments instead of patiently listening to him. Suppose I ask him, towards the end of his spontaneous comments, if when he was complaining about bad listeners last time he was also thinking that I fit into this category. This could be seen both as an interpretation, but also as a case of my priming his memory. Another kind of priming, might be to let him know what I think he seems to be feeling, and ask if this might be the case or not. Or priming could be my mentioning a song that is running through my mind, and either sing it to him or wonder aloud with him if it reflects something in him that I might be tuning in upon. Priming connects past and present feelings in complex ways. ${ }^{15}$ Priming is also the critical mutual activity which connects the minds and brains of two individuals, that is, which puts them in resonance with each other.

Long-term-potentiation (LTP) has been eloquently reviewed by Bliss (1998, pp. 78-82; see also Johnston 1997 and Malenka \& Nicoll 1999), and is likely the precursor of LTM (Bliss 1998). ${ }^{16}$ LTP is defined as the

${ }^{14}$ Like any generalization, my comment about the number of passes required for new implicit memory formation is only relative to explicit memories. Implicit memory formation also requires repetition. Moreover, given our limited present knowledge, it is probably wise not to insist that there are only two major types of memory without overlap, since implicit memory is likely a property of all or at least many neural networks. Recent studies of perceptual learning have further shown clear evidence of V1 modification as a result of training, and it would be surprising that such modification would not occur in many other networks (Michael Posner, personal communication).

${ }^{15}$ For additional information on priming see Schacter \& Buckner 1998.

${ }^{16}$ Bliss states that arguing that LTP is not the basis for LTM brings to mind Mark Twain's remark about those who assert that Homer may not have been the author of the Iliad: "If it wasn't Homer, then it must have been another old blind Greek poet of the same name [!]" (Bliss 1998, p. 91). Incidentally, LTP could also be seen as the variable that relates LTM and neural networks to each other, as that 
easier neural firing secondary to neuronal use or stimulation. LTP is also characterized by so-called specificity and associativity. These are defined as follows:

In terms of the hippocampus, for example, if two sets of converging electrodes are placed on either side of a recording electrode in the CA1 region of the hippocampus, say to activate pyramidal cell axons of the neighboring CA3 region, LTP will be produced in either of the two converging pathways (say CA1 and CA3) providing only that it is active at the time of the stimulating input. In other words, each synapse, in principle acts as an "independent computing device" (this is input sensitivity; Bliss 1998, p. 80). Associativity refers to the ability to induce LTP in the second pathway of the above example, that is, even in the face of a weak tetanus in this pathway, when a strong stimulus is given at the same time to the first pathway. Bliss sees this form of associative memory as coinciding with classical conditioning (Bliss 1998, p. 81). ${ }^{17}$

Each form of memory, implicit or explicit, is also recordable in two phases: short-term and long-term. However, only long-term memory requires the synthesis of new proteins (Albright, Jessell, Kandel \& Posner 2000, p. S13). "Short-term synaptic enhancement [...] results from a covalent modification of preexisting proteins mediated by the cAMPdependent protein kinase A (PKA) and by protein kinase C (PKC)" (Albright et al. 2000, p. S13). In contrast, long-term memory involves "the translocation of PKA and mitogen-activating protein kinase (MAPK) to the nucleus of the sensory neurons where these kinases activate CREB1 and derepress CREB2, leading to the induction of a set of immediate response genes [ERG] and ultimately resulting in the growth of new synaptic connections" [emphasis added] (Bartsch et al. 1995, 1998, cited in Albright et al. 2000, p. S13). ${ }^{18}$ The point here is that at a

activity which leads to "the next step". In other words, other things being equal, LTP leads to neural network formation leads to LTM, more or less.

${ }^{17}$ A further complication touched upon is that something needs to turn off LTP once it has been induced (Bliss 1998, p. 89). This is one aspect of long-term depression (LTD) described most extensively by Ito (2001).

${ }^{18}$ It would seem that the preceding paragraph accurately summarizes a lot of what is known about synapses, at least so far as signal transduction leading towards long-term memory is concerned. However, this is of course an illusion. The real complexity, for example, also would need to include many other subjects, such as lipid rafts. Sphingolipid- and cholesterol-rich domains in the plasma membrane of cells are known as lipid rafts. Within this subcellular domain or compartment proteins (cytokines) are sequestered, and this "increases the specificity and efficiency of signal transduction" (Zacharias, Violin, Newton \& Tsien 2002, p. 913). Also, within 
molecular level all of the known memory systems likely involve roughly the same set of molecular [cytokine] markers (Michael Posner, personal communication). ${ }^{19}$

Returning to the implicit/explicit memory distinction, it is important that in spite of the differences noted, the explicit and implicit memory systems should not be thought of as wholly separate and competitive; rather, they are judged to be highly complementary to each other in the brain and usually create a mosaic of integrated memory formats, i.e. "they ordinarily cooperate with each other in learning" (Squire 1998, p. 68). This is part of the complexity of memory alluded to.

Putting this all together in 1985 Floyd Bloom described the neural cascade, culminating in LTM, as follows: it begins when NE alters the firing of particular neurons by activating cyclic AMP (an intracellular second messenger), which then activates the enzyme protein kinase (noted above), which leads to the phosphorylation of macromolecular substrates (such as pre- and postsynaptic ion channels) and the enhancement of transmission. Obviously, by the mid 1980's, synapses were understood to be fairly complex structures even though the wrinkle of gene activation and CK was less well understood compared to now, and much remained to be discovered.

Contemporary articles on synaptic activity clearly show the growing appreciation and complexity of CK in synaptic changes (and make clear the large number of other cofactors involved - see below on Ito's work). I will mention two in particular. The first is by an American research group, Beattie et al. (2002) which I introduce to make clear the role of glial cells; the second paper, characterizing cerebellar long-term depression (LTD) cerebellum, by Masao Ito (2001) is introduced to explain

these spaces protein-protein interactions "maintain many signaling complexes" (Zacharias et al. 2002, p. 913). We are only gradually learning about the nature of lipid rafts and their controls.

19 The time course of neurotransmitter (cytokine) induced activation of genes makes clear what leads to what: first neurotransmitter binds to receptor, very quickly (within a few minutes) this is followed by the curve of cAMP response, followed by the curve representing the activation of Protein Kinase, then activation of CREB, the activation of ERG and the protein synthesis it leads to (e.g. Fos or Jun) in sequence (Stahl 2000, p. 8, see especially Fig. 2). Curves for late-gene mRNA synthesis, lategene protein synthesis, and long-term effects of late-gene products all follow the previous cascade, in order, as one would expect (i.e. the effects of early genes produce products which can activate late genes starting within 60 minutes or so). 
LTD and critical synaptic subtleties which might otherwise go unnoticed.

The American group describes how "activity-dependent modulation of synaptic efficacy in the brain contributes to neural circuit development and experience-dependent plasticity." They further remark on the decisive role of glial cells as follows: "although glia [supporting cells] are affected by activity and ensheathe synapses, their influence on synaptic strength has largely been ignored" (Beattie et al. 2002, p. 2283).20 The key is that "a protein produced by glia, tumor necrosis factor alpha (TNFalpha) [a CK] enhances synaptic efficacy by increasing surface expression of AMPA receptors" (Beattie et al. 2002, p. 2284) thus playing a role in synaptic plasticity.

To be more precise, however, in spite of explicating the role of glial TNFalpha in activating genes and thus altering AMPA receptors, these researchers did not conclude, at least in their particular set of experiments, that gene activation played a major role in modifying synaptic plasticity; rather, they felt that activity was responsible for the changes in AMPA receptors. Nevertheless, they believe gene activation could not be ruled out absolutely (personal communication). ${ }^{21}$ Obviously, it may not be easy to tell when gene activation is a direct factor in synaptic enhancement, or when indirect. The distinction may not be that critical to our synopsis of learning changes, however.

The complexity of the synapse, as regards CK, emerges still more clearly if we shift to Masao Ito's work (2001). Ito is Director of the Brain Science Institute (BSI) of RIKEN ${ }^{22}$ FRONTIER INSTITUTE in

${ }^{20}$ It has been known that glial cells increase the ability of neurons to form and maintain synapses, by as much as a factor of 7 , but prior to this study, it was not clear how this effect was obtained (Helmuth 2001). A second synapse-promoting signal is apparently the release of cholesterol from glial cells (the work of Mauch et al. 2001). Mauch et al. in turn credit Pfrieger and Barres for their work in 1997 on retinal ganglion cells (Pfrieger \& Barres 1997).

${ }^{21} \mathrm{M}$. Beattie (personal communication) states that he agrees that the effects of TNF alpha on gene expression are very interesting and could be important in the long term regulation of glutamate receptor sensitivity. In the work reported, though, the effects on AMPA receptor surface localization take place within minutes. The previous data from the Malenk/Stanford group and others suggest that there is considerable regulation by modulation of AMPA receptor recycling without (necessarily) the induction of changes in gene expression. Whether longer term effects may be mediated by transcriptional/translational effects remains to be seen."

${ }^{22}$ RIKEN is an abbreviation of "rikagaku kenkyu jo" which in Japanese is Institute for Chemical and Physical [Physics] Research. 
Wako, Japan. If you examine Fig. 2 from Ito's paper LTD, I think you can begin to see the complexity I am talking about. It seems that synaptic phenomena are not only complex, they are probably more complex than most of us can even imagine!

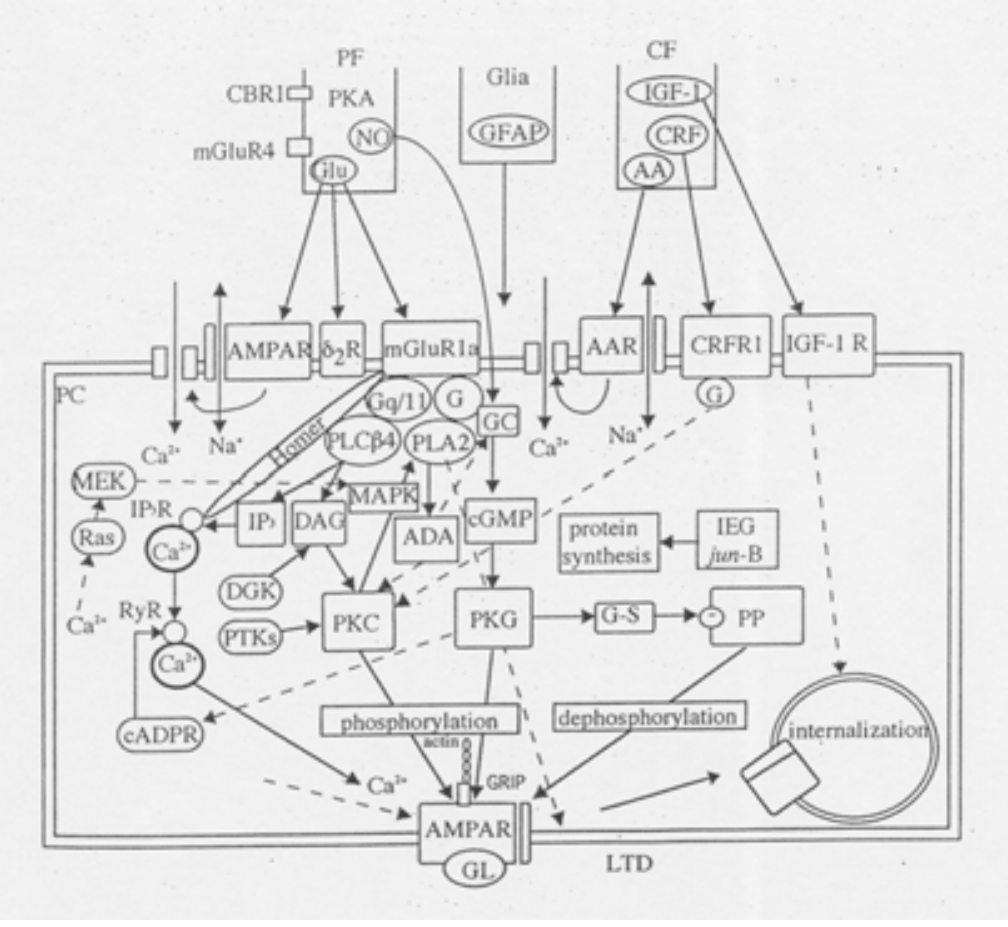

Figure 2

Let me explain some of Fig. 2. It will help to consider the following points:

(1) Ito was early to demonstrate LTD, the experience-based down regulation of neuronal firing (i.e. the opposite of LTP). The required trigger for LTD in the cerebellum is when Purkinje cells are simultaneously fired off (conjunctive stimulation) by numbers of granule cell axons (GA, also abbreviated as PF for parallel fibers), and by single climbing fibers (CF). It is then that the AMPA receptors (AMPAR) of Purkinje cells demonstrate LTD induction. This is illustrated in Fig. 2.

(2) The GA carry the stimuli from basket cells (BC) which carry input from two potential sources, either spinal cord and brain stem, or from the cerebellar flocculus. The CF carry input from the inferior olive or the retina. 
(3) When the input is from the flocculus and retina, the cerebellum is able to provide the vestibular occular reflex (VOR), which compensates for head movements by eye movements to keep retinal targets stable. Ito has done much to understand the basis for the VOR.

(4) In Fig. 2 the upper part of the diagram shows the simultaneous input from PF (i.e. GA), and CF, along with glial input. At the bottom the rectangle stands for the PC, with its AMPAR. Within the rectangle Ito diagrams all the subprocesses, namely (a) the initial processes of signal transduction (including the now familiar first messengers, receptors, ion channels, G proteins, and phospholipases), (b) processes of the transduction itself (including ion concentrations, second messengers, protein kinases and protein phosphotases, activation of the AMPA receptor, and the chemical network for LTD), and, (c) the roles of various other complex factors.

(5) It is important that unlike LTP, in the case of LTD "there is no evidence indicating that adenyl cyclase, cAMP, or PKA has any role in [LTD] induction" (Ito 2001, p. 1155).

At this point I think you can well understand how my initial assertion that CK effect memory and learning, stated as it was without immediate further qualification, came dangerously close to inviting a grossly oversimplified view of the synaptic changes behind memory and learning. Obviously, on the one hand, one needs to be very careful in making a judgment about the nature and degree of CK effects, that is, CK do not operate in isolation. And yet on the other hand, CK-induced gene activation clearly plays a decisive role in synaptic plasticity, especially as regards the establishment of LTM. ${ }^{23}$ What remains for neuroscience to determine is the exact involvement of CK in various types of changes in

${ }^{23}$ In particular, although Ito does not discuss cytokines as a separate topic in his paper, he does include no less than four cytokines within his map of synaptic activity: IGF-I (Insulin-like Growth Factor- I), IEG (immediate early gene/ERG), MAPK (MAP kinases; also called Mitogen-activated protein kinases, or ERK for extracellular signal-regulated kinases), and BDNF (for brain derived neurotrophic factor). Regarding BDNF, it is especially important that recent work shows its ability to provide anterograde stimulation of synapses, something of a surprise (Kohara \& Tsumoto 2002). Postsynaptic neurons "have been thought to release neurotrophic factors which promote extension of axons and differentiation or survival of presynaptic neurons" (Kohara \& Tsumoto 2002), but this transfer is towards the postsynaptic side and it is clearly activity-dependent. This makes clear how we are needing to adjust our oversimplified picture of synapses to meet the new facts. 
synaptic plasticity, and the factors which control these changes, as, for example, Ito has provided par excellence for the cerebellum and LTD.

Before leaving this section let me distill more clearly the significance of Ito's article: As I read it Ito is postulating that the detailed memory mechanisms which he and his coworkers have elaborated for the cerebellum and motor learning in particular might very well apply equally to all implicit learning. As he puts it, there are "functional roles of the cerebellum in implicit memory and learning in general" (Ito 2001, p. 1182). His second important conclusion is that the "investigation of gene regulatory mechanisms for LTD will be of essential importance [emphasis added]. A possibility [also] exists that LTD as functional depression is consolidated as a structural change in synaptic contacts and spines" although he adds "as yet there is no evidence showing morphological changes correlated with LTD" (Ito 2001, p. 1182). Obviously, it will be important to examine each of the elements in the implicit memory system and see which are actionable via gene activation and which by other means.

One additional comment on Ito: having known him personally for a long time I want to remind you if you don't already know it that Ito was the researcher who realized that the cerebellum moves ideas, not just limbs, and is thus deeply involved with learning generally, and not just motor learning. I have often used this idea of his in my own analytic work, especially in the sense of paying attention to the gracefulness of a patient's movements or lack thereof, thinking this can sometimes provide important clues to what the patient is feeling, and where they might be developmentally, but not otherwise communicating except in a kind of pantomime.

I further imagine, that if Loren Eiseley were in treatment with me he would communicate a lot in such pantomime, and it might well be my place to comment upon it, that is, if I could find a non-intrusive way of doing so. This might conjure up in his mind recollections of his interaction with his deaf mother, who was undoubtedly communicating with him in sign language or via gestures, a very special kind of communication that was once quite embarrassing for deaf people to use in public, and for their hearing children as well, although nowadays this situation has greatly improved as sign language has achieved the status of a formal language, on a par with any other. Importantly, if I used my knowledge of sign language to communicate with Eiseley, at the right time, he might respond to me very differently, that is, with more emotional associations than if the same message were communicated by other means 
with less personal associations for him. Of course, this would be another clue that memory is affectively organized.

\section{4 AMPA AND NMDA RECEPTORS}

Summarizing to this point, there are no less than six possible molecular mechanisms that are candidates for explaining the stable synaptic changes we associate with LTM (see Lisman \& Fallon 1999, reviewing the work of Bhalla \& Iyengar 1999, p. 381):

a second messenger switch (in which MAPK activates PKC via PLA2, and where a reverse feedback effect can also occur in which PKC activates MAPK via RAS or RAF);

a kinase switch (in which CAMKII, i.e. CAM-kinase II, autophosphorylates itself once activated by an increase in Calcium ion, a process reversible via phosphatase);

a transcriptional switch (such as our mention of $\mathrm{CK}$ altering gene expression or undoing it);

a translational switch (starting from mRNA which would give rise to a protein and then to a second messenger, where the latter would stabilize a translational switch in the synapse);

a structural unit change associated with turnover within the synapse (such as hinted at in Ito's article);

a structural unit change without turnover (an example being the action of agrin within the neuromuscular junction, a subject peripheral to the present discussion).

We need also to remind ourselves that just as is the case for synapses, AMPA receptors, and other receptors as well, are createable on emotional demand. That is, receptors "are synthesized in the endoplasmic reticulum, processed in the Golgi apparatus, and transported to the cell surface in membrane vesicles [...]to dendrites" as they are needed (Nakagawa \& Sheng 2000, p. 2270). The key to their effectiveness in terms of excitatory synaptic transmission effectively relates to the number of such AMPA receptors that are produced, their rate of production and their exact distribution postsynaptically (Nakagawa \& Sheng 2000; Shi 2001, p. 1851). Using recombinant AMPA receptors Shi has been able to cleverly prove that AMPA receptors are in fact delivered to synapses exactly in relation to LTP induction (Shi 2001). As noted earlier, this decisively proves that there must be a method for communication from the postsynaptic membrane to the presynaptic side, otherwise, how could the presynaptic membrane prepare and deliver receptors in such a timely way? 
The NMDA receptor is also a key player in synaptic plasticity (Ghosh 2002, p. 449). Ghosh, reviewing the work of Takasu, Dalva, Zigmond and Greenberg (2002) points out that "post-synaptic NMDA Calcium ion flux is itself dependent on Eph receptors. Eph receptors are a large family of receptor tyrosine kinases that regulate various developmental events", a reference to the cytokines EphA and EphB (Ghosh 2002; Takasu et al. 2002).

Takasu et al. summarize their findings in the following way: The EphB receptor tyrosine kinases are localized at the NMDA synapses and their role is to effect activity-dependent and activity-independent development and remodeling of synaptic connections (Takasu et al. 2002, p. 491). The importance of such changes in NMDA synaptic reinforcement is that such modifications in the synapses becomes the basis for changes in the hippocampal CA1 region, which is crucial for converting new memories into LTM in the explicit memory system (Shimizu, Tang, Rampon \& Tsien 2000).

\section{LTM: FROM EMOTIONAL ATTENTION TO LTM}

The combined effects of the $C K$ induced cascade of events results in structural changes in the synapse, in changes within the nuclei of neurons, and ultimately the establishment of LTM which grows from larger numbers of such neurons undergoing related changes and operating as parts of neural networks (i.e. ultimately, as databases of mind/brain).

Regarding the creation of LTM, we can summarize again how this likely comes about, based upon knowledge of those specific brain structures most connected with and affected by emotion and how they are activated. Obviously, CK play a role. I have already described the specific $C K$ involved, and at least some of the factors which stimulate $C K$ activity. And as noted above, one key is emotional attention, that is, the changes (chemical, physiological and psychological) which accompany the activity of neural systems and which provide emotional attention, especially the ventral part of the anterior cingulate cortex (ventACC). ${ }^{24}$ Obviously, the ventACC is a critical component in the system being described.

${ }^{24}$ By emotions I am referring not merely to the cortical systems, and of course the limbic system, but also to the deep structures of the brainstem or core, as pointed out by Panksepp (2000, 2001), including the periaqueductal gray (PAG), hypothalamus, etc. 
It will help to briefly review where we have been. As described earlier, LTM "results from the translocation of PKA and mitogen-activated protein kinase (MAPK) to the nucleus of the sensory neurons where these kinases activate CREB1 and derepress CREB2, leading to the induction of a set of immediate response genes and ultimately resulting in the growth of new synaptic connections" (Albright et al. 2000, p. S13, citing the work of Bartsch et al. 1995, 1998).

Ibelgauft, writing under the rubric of CRE (for cyclic AMPresponse element), describes the process as follows: "Cyclic AMPresponse element cAMP second messenger pathways provide a chief means by which cellular growth, differentiation, and function can be influenced by extracellular signals...an inducible enhancer of genes that can be transcribed in response to the increase in cAMP levels" (Ibelgauft 1999, section of CRE, p. 1).

With the notable exception of LTD and thus most implicit learning (which Ito has clarified does not involve adenyl cyclase), cAMP levels need to be enhanced as part of the basic pathway leading to the growth of new synapses and the changing of synaptic plasticity for LTP. So the question then becomes: What can we say about those factors in explicit memory which elevate cAMP in a timely way, since after all this step within the system is what leads to a cascade ending in immediate response genes (IEG, also called ERG for early response genes) and new synaptic production?

Clearly, cAMP can come from a number of sources. If we consider ATP as the source of cAMP level, then one obvious endocrine initiator, as noted in the early article I cited by Floyd Bloom, would have to be $\mathrm{NE}^{25}$ which is known, along with epinephrine, both to break down ATP into 3',5'-cAMP and pyrophosphate, and which also is itself released in response to significant emotional stimulation. In addition, as I noted in discussing the immune part of NIN, sympathetic neurons are sources of $\mathrm{NE}$, and we know that NE is of course also a central neurotransmitter.

Another question remaining is have we tied in the affective systems to the NE release, that is, in relation to affective attention neural network formation, and ultimate LTM formation? The answer seems not hard to

${ }^{25}$ This statement is based on the classical work on Hodgkin, Huxley and Katz (1952), and a minor bit of personal research (Levin 1962) demonstrating that the effects of epinephrine on liver essentially are those induced by 3'5'-AMP (cAMP), which epinephrine produces by catalyzing ATP into cAMP and P-P. NE follows this pattern (Bloom, op. cit.). 
guess. The original affective signal leading to $N E$ release would most likely be received by the ventral division of the anterior cingulate, along with the amygdalar, hippocampal (CA1 cells), and hypothalamic cells in response to emotional activity deeper in the brain's core (e.g. the PAG). It may help to remind ourselves that this ventACC "is connected to the amygdala, PAG, nucleus accumbens, hypothalamus, anterior insula, hippocampus, and orbitofrontal cortex, and has outflow to autonomic [sympathetic], visceromotor and endocrine systems" (Bush, Phan \& Posner 2000, p. 216). Once the NE concentration would rise in association with ventACC, and sufficient cAMP would be available thereby (via the breakdown effect on ATP as described earlier), we would expect the cascade of PKA, MAPK, activation of CREB1 and the deactivation of CREB2 (i.e. in response to shifts in cAMP which effect the kinases, phosphorylation mechanisms, and protein protein interactions of great complexity), ${ }^{26}$ and finally ERG and its effects.

Regarding immediate response genes (ERG), it should be noted that aside from hormones, cAMP signals and cytokines, ERG is triggered by many factors: antigens, neurotransmitters, leukotrienes, prostaglandins, eicosanoids, protein synthesis inhibitors, calcium signals, cellular adhesion molecules, osmotic pressure changes, and stress, to name but a few (Ibelgauft, op. cit., ERG section, p. 1). From this it should be obvious that many other pathways might also play a role in ERG activation in addition to the ones mentioned (viz. via cAMP and NE effects) and thus effect the final stimulus pathway towards formation of LTM. Whatever the contributing factors, however, clearly the brain is designed to provide LTM on demand by multiple mechanisms such as we have been considering.

From the psychoanalytic perspective, the formation of new neural networks and ultimately LTM in association with learning obviously occurs under a variety of circumstances which relate to emotional and attentive processes, including psychoanalytic interventions.

By the way, I believe Loren Eiseley would have been most interested in this paper, especially in the relationship between the affects and

26 "At least ten different CREB protein genes have been cloned, including CREB, CREB1, CREB2, CREBP1, ATF2, ATF (activating transcription factor), ATF43, ATF1, TREB 36. The CREB1 gene maps to human chromosome 2q32.3q34. The human CREB2 gene maps to 2q24.1-q32, a site very close to that of the CREB1 gene. CREB proteins are activated by phosphorylation by cAMP-dependent protein kinase A" (Ibelgauft 1999, CRE section, p. 1). 
ideas generated in his imaginary psychoanalysis, and their associated specific chemical and anatomical changes in his brain. Remember, as a physical anthropologist he often lived mentally at the intersection between the technical chemical and anatomical findings from human artifacts and the history of human stressful experience.

\section{POSTSCRIPT: INTERDISCIPLINARY RESEARCH}

I have just presented some mind/brain details connecting the psychoanalytic and the neuroscientific understanding of learning and memory, at least as this involves learning within the psychoanalytic situation and the on demand effects of such on cytokines and synaptic plasticity. In spite of my effort, however, I suggest that it still remains very difficult to align these two perspectives; to date only a small handful of reliable mind/brain correlations and linkages have been generally accepted as valid. ${ }^{27}$ Only time will tell about the acceptance of the correspondences I have speculated about today. Where then does hope come from for further bridging between mind and brain?

My answer is that our principal hope comes from collaboration among specialists across disciplinary boundaries, and employing various philosophical approaches; only then do we move bit by bit closer to deeper and specific bridgeable understandings within neuroscience and psychoanalysis.

I noted two influential approaches at the beginning: Pragmatism, and what I called thinking things through deeply and creatively. Regarding the former, Stanley Fish states (cited by Arnold Goldberg 2002, p. 249) that "Pragmatism is the philosophy not of grand ambitions but of little steps; and while it cannot help us to take those steps or tell us what they are, it can offer the reassurance that they are possible and more than occasionally." But we also need to respect the creative search for truths independently of what is practical. This seems to me a complementary area where psychoanalysis excels.

Collaboration across disciplinary, cultural, philosophical and language boundaries represents a much needed effort to reduce the complexity of thinking generally by introducing novel testable scientific

${ }^{27}$ In my opinion, credit for the most important mind/brain correlations is particularly due Jaak Panksepp (1998, 2000, 2001), and to Mark Solms and Karen Kaplan Solms, some of which appears in the latter's book (Kaplan-Solms \& Solms 2000) or in the pages of Neuro-Psychoanalysis (Solms 1999). 
paradigms, as suggested by Giampieri-Deutsch (personal communication). In the future, progress will not be accomplished without larger groups of specialists working together.

The Pragmatism I refer to has been recently explicated by Goldberg $(2002)^{28}$. Practical approaches appeal whenever the subject matter of research interest reaches a critical level of complexity. This is surely the case for our current interest in studying mind or brain both in depth and in tandem. On the other hand, as suggested by Gedo (personal communication), there is also a place for creative thinking in other modes where, for example, discovering the deepest truths is the primary consideration. Combining the search for deep truths with practical real time experimentation and verification may be the best of both worlds.

When we recognize the limitations of our current knowledge, and the obvious benefits of collaborating across disciplinary boundaries, both personally and professionally, we gain in strength, and slowly expand our horizons about such phenomena as memory, emotions, and learning. Therefore, even though we cannot be entirely sure in what way emotional signals emanate from the mind/brain and serve as a critical trigger for the creation of new hope and useful knowledge, we cannot afford to give up our efforts towards eventually discovering more of their technical details. These details obviously lie in our describing more clearly as many as possible of our current unclarities (die "Unklarheiten"), which I hope you will agree I have succeeded in presenting to you. Only then does Night become brighter, as when in analysis we do our best to connect with a fellow sufferer in a compassionate way, and hope this helps him or her also better connect with themselves.

${ }^{28}$ Incidentally, since my reference to Pragmatism may undoubtedly confuse some readers, let me briefly expand on what I intend here. I am especially thinking of Charles Sanders Pierce's original thinking about Pragmatism, that is, that the basis of truth determination is via experimental pragmatic results, and I am much less inclined towards William James' and others later modification of Pierce's intentions in the direction of pragmatic subjectivity, or mere efficacy (see Rescher 1995). I also intend the idea that scientists make critical practical decisions in the discovery phase of any investigation, which involves deciding, for example, how to divide complex problems into subproblems that will yield more easily to experimental study. 


\section{REFERENCES}

Abel, T., Martin, K.C., Bartsch, D. \& Kandel, E.R. (1998). Memory suppresser genes: Inhibitory constraints on the storage of long-term memory. Science 279: 333-341.

Ahlquist, P. (2002). RNA-dependent RNA polymerase, viruses, and RNA silencing. Science 296: 1270-1273.

Albright, T.D., Jessell, T.M., Kandel, E.R. \& Posner, M.I. (2000). Neural science: A century of progress and the mysteries that remain. Cell 10: S1-S55.

Bartsch, D., Ghirardi, M., Skehel, P.A., Karl, K.A., Herder, S.P., Chen, M., Bailey, C.H. \& Kandel, E.R. (1995). Aplysia CREB2 represses long-term facilitation: relief of repression converts transient facilitation into long-term functional and structural change. Cell 83: 979-992.

Bartsch, D., Casadio, A., Karl, K.A., Serodio, P. \& Kandel, E.R. (1998). CREB1 encodes a nuclear activator, a repressor, and a cytoplasmic modulator that form a regulatory unit critical for long-term facilitation. Cell 95: 211-223.

Beattie, E.C., Stellwagen, D., Morishita, W., Breshahan, J.C., Ha, B.K., von Zastrow, M., Beattie, M.S. \& Malenka, R.C. (2002). Control of synaptic strength by glial TNFalpha. Science 295: 2282-2285.

Bhalla U.S. \& Iyengar R. (1999). Emergent properties of networks of biological signaling pathways. Science 283: 381-7.

Bliss, T. (1998). The physiological basis of memory. In From Brains to Consciousness?: Essays on the New Sciences of the Mind, ed. S. Rose. Princeton: Princeton University Press, pp. 73-93.

Bloom, F.E. (1985). CNS plasticity: A survey of opportunities, In Central Nervous System Plasticity and Repair, ed. A. Bignami, A. Adoloye, F.E. Bloom \& C.L. Bolis. New York: Raven Press, pp. 3-51.

Bush, G., Phan, L. \& Posner, M.I. (2000). Cognitive and emotional influences in anterior cingulate cortex. Trends in Cognitive Sciences 4: 215-222.

Castillo, P.E., Schoch, S., Schmitz, F., Südhof, T.C. \& Malenka, R.C. (2002). RIM1alpha is required for presynaptic long-term potentiation. Nature 415: 327330 .

Contractor, A., Rogers, C., Maron, C., Henkemeyer, M., Swanson, G.T. \& Heinemann, S.F. (2002). Trans-synaptic Eph receptor-ephrin signaling in hippocampal mossy fiber LTP. Science 296: 1864-1869.

Csikszentmihalyi, M. (1975). Beyond Boredom and Anxiety: The Experience of Play in Work and Games. San Francisco: Jossey-Bank.

Dobrunz, L.E. \& Garner, C.C. (2002). Priming plasticity. Nature 415: 277-278.

Eiseley, L. (1971). The Night Country. New York: Charles Scribner's Sons/Macmillan Publ. Co.

Fonagy, P. (1998). Moments of change in psychoanalytic theory: Discussions of a new theory of psychic change. Infant Mental Health Journal 19: 346-353.

Ghosh, A. (2002). Learning more about NMDA receptor regulation. Science 295: $449-451$.

Goldberg, A. (2002). American pragmatism and American psychoanalysis. Psychoanalytic Quarterly 71: 235-250. 
Gould, E., Reeves, A.J., Graziano, M.S. \& Gross, C.G. (1999). Neurogenesis in the neocortex of adult primates. Science 286: 548-552.

Hellmuth, L. (2001). Glia tell neurons to build synapses. Science 291: 569-570.

Hodgkin, A.L., Huxley, A.F. \& Katz, B. (1952). Measurement of current-voltage relations in the membrane of the giant axon in Loligo. Journal of Physiology 116: 424-448.

Ibelgauft, H. (1999). Cytokines Online Pathfinder Encyclopedia. $\mathrm{http}: / /$ www.copewithcytokines.de.

Ito, M. (2001). Cerebellar long-term depression: characterization, signal transduction, and functional roles. Physiological Reviews 81: 1143-1195.

Johnston, D. (1997). A missing link? LTP and learning. Science 278: 401-402.

Kaplan-Solms, K. \& Solms, M. (2000). Clinical Studies in Neuro-Psychoanalysis: An Introduction to a Depth Neuropsychology. New York: Karnac Books.

Khan, A.A., Bose, C., Lung, S.Y., Soloski, M.J. \& Rupp, F. (2001). Physiological regulation of the immunological synapse by agrin. Science 292: 1681-1686.

Kohara, K. \& Tsumoto, T. (2001). Activity-dependent transfer of brain-derived neurotrophic factor to postsynaptic neurons. Science 291: 2419-2423.

Kohara, K. \& Tsumoto, T. (2002). Annual Report of Osaka University: Subreport of Research Activities. http://www.osakau-u.ac.jp/eng/research/graphics/.

Levin, F.M. (1962). The effect of epinephrine and 3'5'AMP on the perfused rabbit liver. The Physiologist 5: 175.

Levin, F.M. (1991). Mapping the Mind: The Intersection of Psychoanalysis and Neuroscience. Hillsdale: The Analytic Press.

Levin, F.M. (1995). Psychoanalysis and knowledge. Part 1: The problem of representation and alternative approaches to learning. The Annual of Psychoanalysis 23: 95-114.

Levin, F.M. (1997). Integrating some mind and brain views of transference: the phenomena. Journal of the American Psychoanalytic Association 45: 11211151.

Levin, F.M. (2002). The neuroimmune network and its relevance to psychoanalysis. Psychoanalytic Quarterly 71 (3).

Levin, F.M. (2003). Psyche and Brain: The Biology of Talking Cures. Madison: International Universities Press.

Linden, D.J. \& Routtenberg, A. (1989). The role of protein kinase C in long-term potentiation: a testable model. Brain Research Reviews 14: 279-296.

Lisman, J.E. \& Fallon, J.R. (1999). What maintains memories? Science 283: 339340.

Maier, S.F. \& Watkins, L.R. (1998). Cytokines for psychologists: Implications of bidirectional immune-to-brain communication for understanding behavior, mood, and cognition, Psychological Review 105: 83-107.

Malenka, R.C. \& Nicoll, R.A. (1999). Long-term potentiation - A decade of progress? Science 285: 1870-1874.

Mauch, D.H., Nägler, K., Schumacher, S., Göritz, C., Müller, E.-C., Otto, A. \& Pfrieger, F.W. (2001). CNS synaptogenesis promoted by glia-derived cholesterol. Science 294: 1354-1357.

Nakagawa, T. \& Sheng, M. (2000). A stargazer foretells the way to the synapse. Science 290: 2270-2271. 
Panksepp, J. (1998). Affective Neuroscience: The Foundation of Human and Animal Emotions. Oxford, New York: Oxford University Press.

Panksepp, J. (2000). The neurodynamics of emotions: An evolutionaryneurodevelopmental view, In Emotion, Self-Organization, and Development, ed. M.D. Lewis \& I. Granic. New York: Cambridge University Press, pp. 236264.

Panksepp, J. (2001). The neuro-evolutionary cusp between emotions and cognitions. Evolution and Cognition 7: 141-163.

Pfrieger F.W. \& Barres, B.A. (1997) Synaptic efficacy enhanced by glial cells. Science 277: 1684-1687.

Rescher, N. (1995). Pragmatism. In The Oxford Companion to Philosophy, ed. T. Honderich. Oxford, New York: Oxford University Press, pp. 710-713.

Schacter, D.L. \& Buckner, R.L. (1998). Priming and the brain. Neuron 20: 1-20.

Schoch, S., Castillo, P.E., Jo, T., Mukherjee, K., Geppert, M., Wang, Y., Schmitz, F., Malenka, R.C. \& Südhof, T.C. (2002). RIM 1 alpha forms a protein scaffold for regulating neurotransmitter release at the active zone. Nature 415: 321-326.

Shi, S.-H. (2001). AMPA receptor dynamics and synaptic plasticity. Science 294: 1851-1852.

Shimizu, E., Tang, Y.-P., Rampon, C. \& Tsien, J.Z. (2000). NMDA receptordependent synaptic reinforcement as a crucial process for memory consolidation. Science 290: 1170-1173.

Solms, M. (1999). The new neuropsychology of sleep. Neuro-Psychoanalysis 1: $183-195$.

Squire, L. (1998). Memory and brain systems. In From Brains to Consciousness?: Essays on the New Sciences of the Mind, ed. S. Rose. Princeton: Princeton University Press, pp. 53-72.

Stahl, S.M. (2000). Molecular neurobiology for practicing psychiatrists. Part 5: How a leucine zipper can turn on genes - immedite-early genes activate late-gene expression in the brain. Journal of Clinical Psychiatry 61: 7-8.

Takasu, M.A., Dalva, M.B., Zigmond, R.E. \& Greenberg, M.E. (2002). Modulation of NMDA receptor-dependent calcium influx and gene expression through EphB receptors. Science 295: 491-495.

Wilson, R.I. \& Nicoll, R.A. (2002). Endocannabinoid signaling in the brain. Science 296: 678-682.

Zacharias, D.A., Violin, J.D., Newton, A.C. \& Tsien, R.Y. (2002). Partitioning of lipid-modified monomeric GFPs into membrane microdomains of live cells. Science 296: 913-916.

\section{FIGURE CAPTIONS}

Figure 1. A simplified scheme of the hypothalamic-pituitary axis (HPA). IS and CNS are captured in a snapshot that shows as well a bit of the complexity of the NIN (Ibelgauft 1999).

Figure 2. Signal transduction underlying LTD (Ito 2001, p. 1151). 
C. Empirical Research

Trends in Psychoanalysis 
